\title{
EDITORIAL
}

\section{Respiratory Morbidity in Late-Preterm Infants: Prevention Is Better Than Cure!}

\begin{abstract}
At a recent meeting of obstetricians and neonatologists, I asked the audience what, in its opinion, was the source of fetal lung fluid. To my surprise, a good number of practitioners were comfortable with the traditional concept that amniotic fluid is an extension of, and a major contributor to, the fluid occupying fetal lungs. When asked if fetal lung maturity testing accurately predicted the risk of respiratory distress in the newborn, a large number said that a mature surfactant profile meant there was little or no risk to the fetus, hence the comfort with delivering the fetus at less-than-term gestation. And finally, when asked if patients have a good sense of the risks and benefits of early delivery when maternal or fetal indications for elective delivery are not as clearcut, all I heard from the audience was, well, a rumble!

Why is it that nearly 30 years after the discovery that fetal lungs actively secrete lung fluid, and more than a decade after the epithelial sodium channel responsible for clearance of the fluid during neonatal transition was cloned, misconceptions abound about one of the most important events in the life of an infant? These concerns have been further underscored by the recent rise in the number of infants born at late-preterm (34 0/7 to 36 6/7 weeks) gestations and the recognition that they carry a higher risk of morbidity and death. ${ }^{1,2}$

For a proper understanding of the reasons behind this increased vulnerability, it is important to understand the physiological events that facilitate transition of the neonate to air breathing. Through much of the gestation, fetal lungs actively secrete fluid into alveolar spaces via a chloride secretory mechanism that can be blocked by inhibitors of $\mathrm{Na}-\mathrm{K}-2 \mathrm{Cl}$ co-transport. ${ }^{3,4}$ This body of fluid plays a critical role in lung development, providing a structural template that prevents collapse of the developing lung and promotes its growth. Conditions that interfere with normal production of lung liquid, such as pulmonary artery occlusion, diaphragmatic hernia, and uterine compression of the fetal thorax from chronic leak of amniotic fluid, are also known to inhibit lung growth. ${ }^{5}$
\end{abstract}

After birth, for effective gas exchange to occur, the fluid must be rapidly cleared from potential airspaces. This process is much more complicated than what the traditional "vaginal squeeze" or Starling forces would suggest. $^{6}$ In fact, much before the onset of spontaneous labor, the fetus begins its preparation for smooth landing by changing its hormonal milieu and by reducing the rate of lung fluid secretion. ${ }^{7}$ After birth, the remaining fluid is rapidly cleared by a two-step process (Fig. 1). The first step is passive movement of $\mathrm{Na}^{+}$from the lumen across the apical membrane into the cell through $\mathrm{Na}^{+}$-permeable ion channels; the second step involves active extrusion of $\mathrm{Na}^{+}$from the cell across the basolateral membrane into the serosal space. ${ }^{7}$ Epithelial $\mathrm{Na}^{+}$ channels $(\mathrm{ENaC})$, which regulate the first step, are rate limiting in this process and developmentally timed for maximum expression only in late gestation. Steroids play a key role in this transition and thus in the absorption of fetal lung fluid. ${ }^{8}$

So why is this discussion of fetal lung fluid relevant to the late-preterm infant, and to the article by Ventolini et $\mathrm{al}^{9}$ highlighted in this issue of the journal? First, Ventolini et al confirm what neonatologists have recognized all along: Birth at 34 to 36 weeks of gestation is a risky proposition because of the high risk of respiratory morbidity in the neonate. However, what is unsettling in their observations is the magnitude of respiratory distress; even more striking is the inordinately high risk of admission to the neonatal intensive care unit (NICU). Second, the issue of surfactant maturity at these gestations notwithstanding, exposure to antenatal steroids in the weeks prior to birth at $340 / 7$ to $366 / 7$ weeks is associated with a striking decrease in respiratory distress $(24.4 \%$ in those exposed to steroids, $81.3 \%$ in those with no steroid exposure; $p<0.0001$ ). There was a similar decrease in respiratory distress syndrome (surfactant deficiency), albeit to a lesser degree (7.5\% in the steroid-exposed group versus $35.5 \%$ in the no-steroid group). These observations bring us back to where we started, which is that not all respiratory distress
${ }^{1}$ Department of Pediatrics and Physiology, Emory University School of Medicine, Atlanta, Georgia.

Address for correspondence and reprint requests: Lucky Jain, M.D., Professor of Pediatrics, Executive Vice Chairman, Emory University School of Medicine, 2015 Uppergate Drive, Atlanta, GA 30322.
Am J Perinatol 2008;25:75-78. Copyright (C) 2008 by Thieme Medical Publishers, Inc., 333 Seventh Avenue, New York, NY 10001, USA. Tel: +1(212) 584-4662.

Accepted: November 29, 2007. Published online: January 23, 2008. DOI 10.1055/s-2007-1022471. ISSN 0735-1631. 


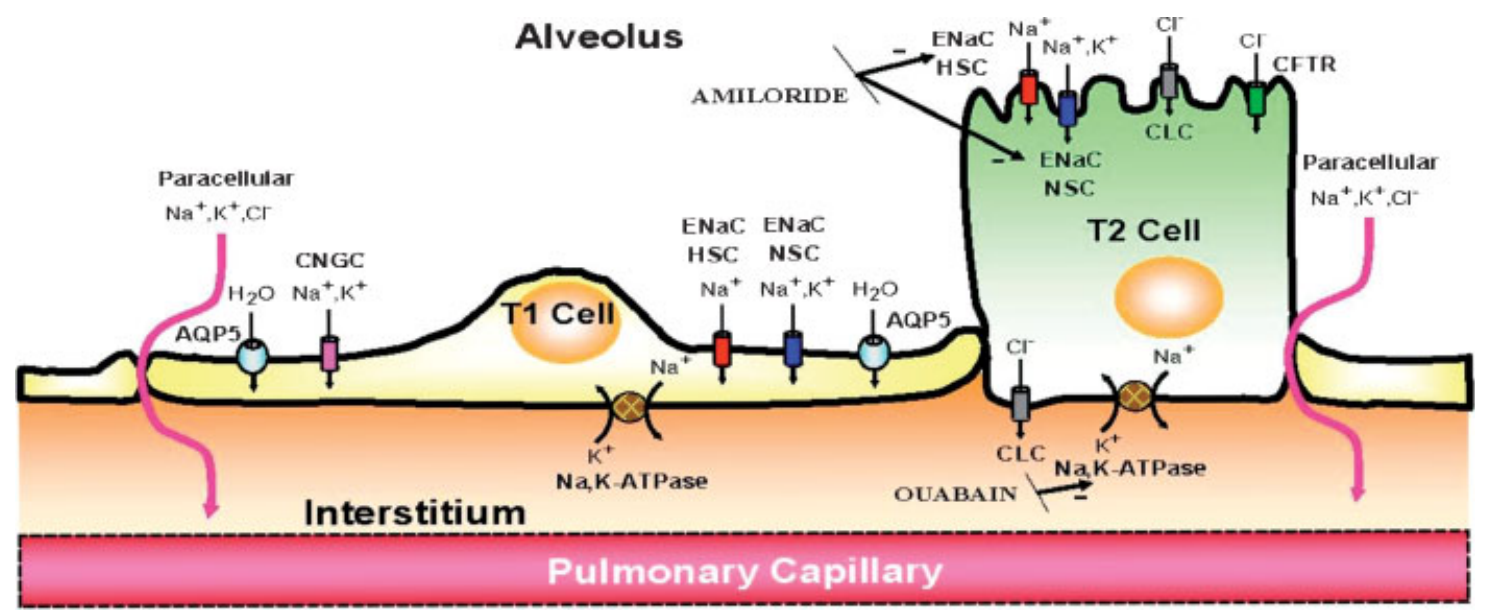

Figure 1 Epithelial sodium ( $\mathrm{Na}$ ) absorption in the fetal lung near birth. Na enters the cell through the apical surface of both ATI and ATII cells via amiloride-sensitive epithelial $\mathrm{Na}$ channels (ENaC), both highly selective channels (HSC) and nonselective channels (NSC), and via cyclic nucleotide gated channels (seen only in ATI cells). Electroneutrality is conserved with chloride movement through cystic fibrosis transmembrane conductance regulator (CFTR) or through chloride channels (CLC) in ATI and ATIl cells, and/or paracellularly through tight junctions. The increase in cell Na stimulates Na-K-ATPase activity on the basolateral aspect of the cell membrane, which drives out three $\mathrm{Na}$ ions in exchange for two $\mathrm{K}$ ions, a process that can be blocked by the cardiac glycoside ouabain. If the net ion movement is from the apical surface to the interstitium, an osmotic gradient would be created, which would in turn direct water transport in the same direction, either through aquaporins or by diffusion.

in preterm infants is due to surfactant deficiency, and steroids may have an impact on both surfactant maturity and fetal lung fluid clearance.

There are limitations to the data that Ventolini et al present. First, the retrospective nature of their data limits the conclusions that can be drawn from it. Data was not available for the timing of steroid administration in mothers who were treated for preterm labor before their delivery at 34 to 36 weeks of gestation, hence the inability to draw conclusions about the duration of steroid exposure required for clinical efficacy. Second, it is not clear why the overall NICU admission rate and risk of respiratory distress is so high in this cohort. It is possible that NICU admissions in the reporting centers were dictated by protocols driven by weight and gestational age rather than by the clinical picture alone. The authors were unable to sort out this issue in their database.

How, then, should we weigh the risks and benefits of electively delivering a fetus early (especially for an indication that may benefit the mother but not the infant), and how do we evaluate the pros and cons of a preventive strategy (like antenatal steroids)? A good starting point would be to critically evaluate the morbidity and mortality burden associated with late prematurity. Concern about higher morbidity in latepreterm infants is reflected in several recent publications, with largely the same conclusions: late-preterm infants are more prone to problems related to delayed transition and overall immaturity, and they should therefore be treated differently than their more mature full-term counterparts. ${ }^{2,10-13}$ Nearly $50 \%$ of infants born at 34 weeks of gestation are admitted to NICUs.
These observations have led to greater attention being paid to tracking short-term morbidity, health care costs, hospital stays, and issues such as rehospitalization. ${ }^{13}$ However, widespread publicity has yet to make a measurable impact on the incidence of late prematurity; nearly three out of four preterm births occur at latepreterm gestations, and this number is on the rise. ${ }^{14} \mathrm{It}$ is estimated that nearly 250,000 late-preterm births occurred in the United States in 2004, and although the problem appears to be widespread, similar estimates from other nations are not readily available. The broad range of neonatal complications that have been reported include delayed lung fluid clearance (transient tachypnea of the newborn), respiratory distress syndrome, pulmonary hypertension, apnea, temperature instability, hypoglycemia, jaundice, and poor feeding. ${ }^{12,14}$ Little is known, however, about the longterm impact of these "transitional issues" because there are no data repositories with information about outcomes, and in spite of growing concern about the vulnerability of the late-preterm brain to white matter injury, systematic developmental assessments are seldom performed. Recent reports about the occurrence of serious complications such as hypoxic respiratory failure and kernicterus are good first steps; compilation of accurate mortality statistics would be another. ${ }^{11,15}$ A recent article by Tomashek et $\mathrm{al}^{16}$ highlighted differences in mortality between late-preterm and term infants. Using period-linked birth-infant death files from 1995 to 2002, the authors analyzed overall and cause-specific mortality rates for singleton late-preterm and term infants. The authors report that although significant declines in mortality were observed over the 
last decade for both groups of infants, the infant mortality rate for late-preterm infants was several-fold higher than that for term infants. Late-preterm infants were particularly more likely to die in the early neonatal period compared with term infants from causes such as respiratory compromise, maternal complications of pregnancy, and congenital anomalies.

What about long-term outcomes? As would be expected of a problem that has hit our radar screens only recently, few long-term data are available about the impact of late prematurity on brain development and neurological outcome. Two recent studies deserve mention, though, because they highlight the seriousness of this issue. Kinney et $\mathrm{al}^{17}$ have shown that only $65 \%$ of the brain volume is accrued at 34 weeks, and in this stage of rapid brain growth, the late-preterm brain may be particularly vulnerable to white matter injury. To highlight this point, the authors evaluated brain specimens at autopsy of late-preterm infants who died in the neonatal period and found a strikingly high incidence of periventricular leukomalacia. This is the same kind of lesion that has received a lot of attention in very low birthweight infants for its high correlation with long-term neurological deficits, particularly spastic diplegia. A more recent study from Sweden reports that among a cohort of $\sim 500,000$ adults in their 20 s, $74 \%$ of the neurologically disabled individuals were born between 33 and 38 weeks of gestation. ${ }^{18}$ These retrospective reports, of course, in no way confirm causality. It is not clear if the neurological injury resulted from or predated the event that caused the late-preterm birth. However, the hypotheses generated by these reports now need further testing; namely, are late-preterm infants at higher risk for short- and long-term morbidity and death than their term counterparts, and if so, why?

In spite of the growing recognition of the disease burden, and the fact that some of it may be preventable, the obstetric community has yet to fully embrace the public health impact of late prematurity. The mean gestational age at birth in the United States has steadily decreased over the last decade from 40 to 39 weeks, and the rate of spontaneous and medically indicated births at $340 / 7$ to 36 6/7 weeks of gestation has increased by 12 to $13 \%$ each. ${ }^{14}$ Late-preterm infants are considered functionally mature (hence the widespread use of the "near term" label), and there is a relative lack of attention to neonatal considerations when delivery at these gestations is being contemplated. Although women in preterm labor at gestations of $\leq 33$ weeks are routinely considered for tocolysis and antenatal steroids, they are candidates for neither if gestation has advanced by a few days and crosses over to the magical 34-week mark. These decisions appear oblivious to the fact that inaccuracies in the estimation of gestational age abound, and up to $50 \%$ of infants at 34 weeks of gestation may require intensive care. ${ }^{10}$
So what will it take to drive a concerted effort to tackle this problem? A good starting point will be the availability of reliable data about short- and long-term outcome of late-preterm infants and documentation of serious morbidity that could dispel the myth of the "transient" nature of late-preterm woes. The findings reported by Ventolini et al and others should also foster debate around the rationale for preterm delivery, particularly when the decision to do so is based on soft indications. Although the studies provide no direct link to the widespread practice of induction of labor and/or elective cesarean deliveries, it raises questions about the recent rise in such practices, particularly in the face of uncertainty in accurate estimation of gestational age. The "discretionary" preterm deliveries deserve a thorough review of the risks and benefits of continuing the pregnancy to both the mother and her infant. Finally, the study by Ventolini et al underscores the urgent need for prospective studies to study strategies that can enhance maturity of the late-preterm fetus, such as the use of antenatal steroids. For a low-cost and lowtech intervention such as two intramuscular injections, and a safety track record that spans nearly three decades, the time to evaluate the role of antenatal steroids in deliveries of 34 to 37 weeks of gestation has come. For the late-preterm gestations, prevention may indeed be better than cure!

\section{ACKNOWLEDGMENTS}

This work was supported by National Institutes of Health Grant R01HL-063306.

$$
\text { Lucky Jain, M.D. }{ }^{1}
$$

\section{REFERENCES}

1. Jain L. Morbidity and mortality in late-preterm infants: more than just transient tachypnea! J Pediatr 2007;151(5):445446

2. Raju TN, Higgins RD, Stark AR, Leveno KJ. Optimizing care and outcome for late-preterm (near-term) infants: a summary of the workshop sponsored by the National Institute of Child Health and Human Development. Pediatrics 2006;118(3): 1207-1214

3. Jain L, Eaton DC. Physiology of fetal lung fluid clearance and the effect of labor. Semin Perinatol 2006;30(1):3443

4. Bland RD. Lung epithelial ion transport and fluid movement during the perinatal period. Am J Physiol 1990;259(2 Pt 1): L30-L37

5. Wallen LD, Kulisz E, Maloney JE. Main pulmonary artery ligation reduces lung fluid production in fetal sheep. J Dev Physiol 1991;16(3):173-179

6. Bland RD. Loss of liquid from the lung lumen in labor: more than a simple "squeeze." Am J Physiol Lung Cell Mol Physiol 2001;280(4):L602-L605 
7. Jain L. Alveolar fluid clearance in developing lungs and its role in neonatal transition. Clin Perinatol 1999;26(3):585599

8. Jain L, Chen XJ, Ramosevac S, Brown LA, Eaton DC. Expression of highly selective sodium channels in alveolar type II cells is determined by culture conditions. Am J Physiol Lung Cell Mol Physiol 2001;280(4):L646-L658

9. Ventolini G, Neiger R, Mathews L, et al. Incidence of respiratory disorders in neonates born between 34 and 36 weeks of gestation following exposure to antenatal corticosteroids between 24 and 34 weeks of gestation. Am J Perinatal 2008; 25(2):79-84

10. Dudell GG, Jain L. Hypoxic respiratory failure in the late preterm infant. Clin Perinatol 2006;33(4):803-830; abstract viii-ix

11. Jain L, Dudell GG. Respiratory transition in infants delivered by cesarean section. Semin Perinatol 2006;30(5):296-304

12. Wang ML, Dorer DJ, Fleming MP, Catlin EA. Clinical outcomes of near-term infants. Pediatrics 2004;114(2):372376
13. Escobar GJ, Greene JD, Hulac P, et al. Rehospitalisation after birth hospitalisation: patterns among infants of all gestations. Arch Dis Child 2005;90(2):125-131

14. Davidoff MJ, Dias T, Damus $K$, et al. Changes in the gestational age distribution among U.S. singleton births: impact on rates of late preterm birth, 1992 to 2002. Semin Perinatol 2006;30(1):8-15

15. Clark RH. The epidemiology of respiratory failure in neonates born at an estimated gestational age of 34 weeks or more. J Perinatol 2005;25(4):251-257

16. Tomashek KM, Shapiro-Mendoza CK, Davidoff MJ, Petrini JR. Differences in mortality between late-preterm and term singleton infants in the United States, 1995-2002. J Pediatr 2007;151(5):450-456

17. Kinney HC. The near-term (late preterm) human brain and risk for periventricular leukomalacia: a review. Semin Perinatol 2006;30(2):81-88

18. Lindstrom K, Winbladh B, Haglund B, Hjern A. Preterm infants as young adults: a Swedish national cohort study. Pediatrics 2007;120(1):70-77 